\title{
Infiltrative hepatocellular carcinoma with multiple lung metastasis com- pletely cured using nivolumab: a case report
}

\author{
Ji Eun Han, Hyo Jung Cho, Soon Sun Kim, Jae Youn Cheong \\ Department of Gastroenterology, Ajou University School of Medicine, Suwon, Korea
}

Received Jun. 5, 2021

Revised Aug. 5, 2021

Accepted Aug. 26, 2021
The current Food and Drug Administration-approved systemic treatments for advanced hepatocellular carcinoma (HCC) include multikinase inhibitors (tyrosine kinase inhibitor [TKI]) and immune checkpoint inhibitors (ICls). Among ICls, nivolumab is used as secondline therapy for advanced HCC after sorafenib failure or patient intolerance. In this case, a patient with infiltrative HCC and portal vein tumor thrombosis was treated with hepatic arterial infusion chemotherapy (HAIC) and radiation therapy. New lung metastasis developed after HAICs; thus, lenvatinib treatment was initiated. However, the disease progressed. Thereafter, sorafenib treatment was initiated but he developed intolerance, with grade 3 sorafenib-related diarrhea. Subsequently, nivolumab was administered as rescue therapy. He demonstrated a partial response to nivolumab after the third treatment and viable HCCs in the lungs and liver completely disappeared after the 24th treatment. These findings suggest that nivolumab could be used as an effective rescue therapy for advanced HCC progression after TKI treatment. (J Liver Cancer 2021;21:169-176)

Keywords: Nivolumab; Immune checkpoint inhibitor; Advanced hepatocellular carcinoma; Case report

\section{INTRODUCTION}

Hepatocellular carcinoma (HCC) is the fourth most common cause of cancer-related mortalities worldwide. HCC originates from various etiologies, such as hepatitis B virus (HBV), hepatitis C virus, and alcoholic and non-alcoholic fatty liver, and usually develops from advanced chronic liver disease or cirrhosis. HCC is often accompanied by the deterioration of hepatic function or portal vein tumor thrombosis (PVTT) ${ }^{1-3}$ Moreover, HCC undergoes multi-step genetic

\section{Corresponding author: Hyo Jung Cho}

Department of Gastroenterology, Ajou University School of Medicine, 164 Worldcup-ro, Yeongtong-gu, Suwon 16499, Korea

Tel. +82-31-219-6939, Fax. +82-31-219-7820

E-mail: pilgrim8107@hanmail.net modification as it progresses. ${ }^{4}$ Subsequently, it exhibits intratumor heterogeneity, does not respond to specific molecular targeted therapy, and often exhibits poor prognosis, especially when accompanied by PVTT or extrahepatic metastases.

Various HCC guidelines recommend sorafenib and lenvatinib as Food and Drug Administration (FDA)-approved multi-tyrosine kinase inhibitors (TKIs) for the first-line treatment of advanced HCC. Recently, immune checkpoint inhibitors (ICIs), which target programmed cell death 1 protein or programmed death-ligand 1 (PD-L1), have also been used as a systemic treatment for advanced HCC following the demonstration of their high efficacy and safety through various clinical trials.

Based on the results of the CheckMate-040 trial, nivolumab, a monoclonal antibody against PD-1, was approved by 
the FDA in 2017 as a second-line therapy for patients with advanced HCC that progressed even after sorafenib treatment or sorafenib intolerance. ${ }^{5}$ CheckMate-040 was an open-label multicenter clinical trial involving 154 patients with advanced HCC that progressed even after sorafenib treatment or due to sorafenib intolerance. In this trial, nivolumab treatment after sorafenib failure exhibited durable response, prolonged overall survival, and manageable safety profile.

Here, we report a case of advanced HCC with PVTT and multiple lung metastases, which demonstrated a complete response (CR) to nivolumab administered after the failure of hepatic artery infusion chemotherapy (HAIC), and lenvatinib, and sorafenib treatments. This case report is described according to the CARE guidelines (https://www.care-statement.org/).

\section{CASE REPORT}

A 56-year-old male patient was referred from a local clinic to our hospital for suspected infiltrative HCC with PVTT occupying the entire sixth segment of his liver, as observed on computed tomography (CT) and magnetic resonance imaging (MRI). He was also diagnosed with chronic hepatitis B and liver cirrhosis for the first time.

Liver MRI showed that approximately $10 \mathrm{~cm}$ of the heterogeneous mass was partially enhanced, with most of the mass exhibiting high signal intensity in the delayed arterial phase.
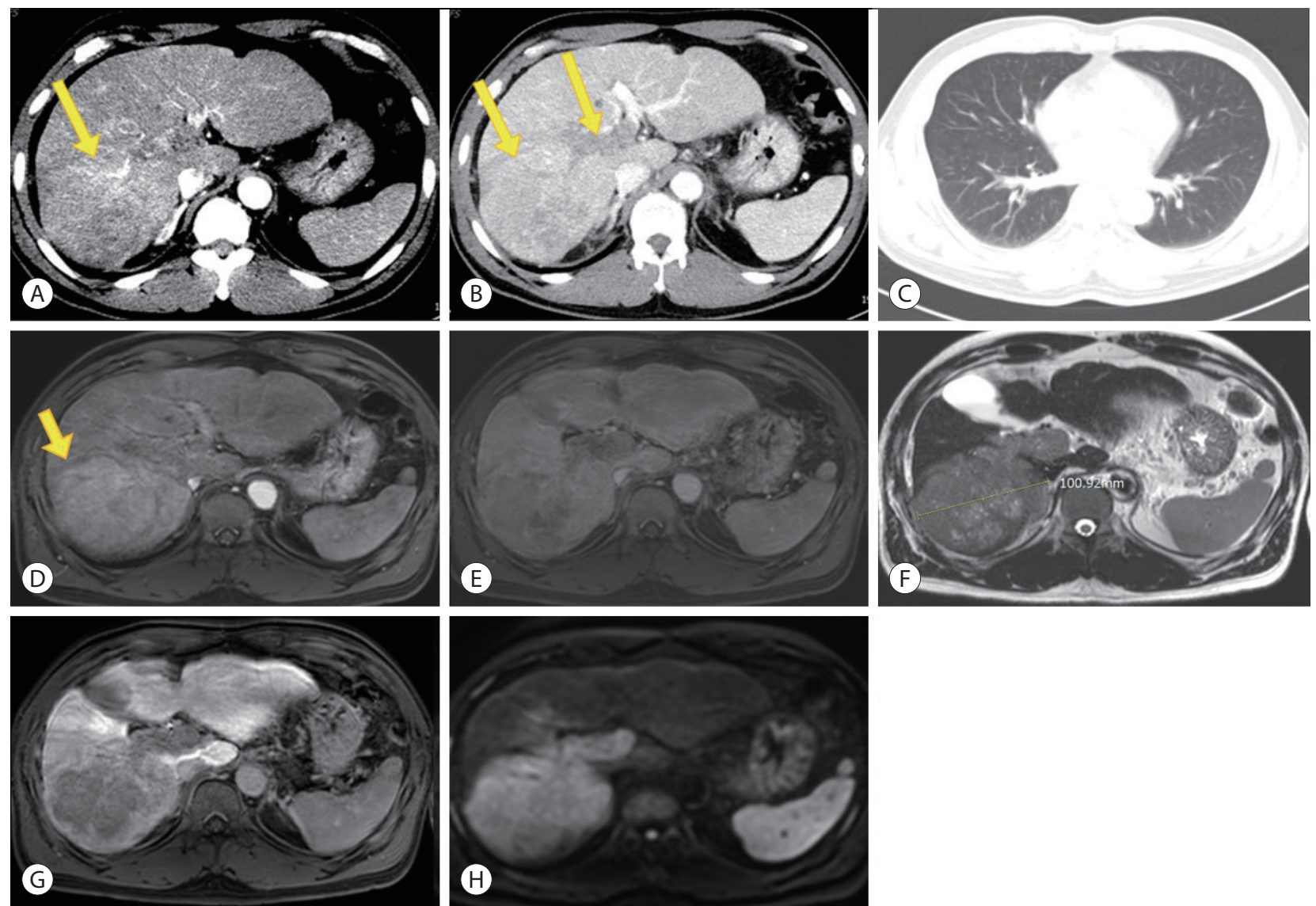

Figure 1. Hepatocellular carcinoma at initial diagnosis in order of abdomen, chest computed tomography (CT) and liver magnetic resonance imaging (MRI). Abdominal CT showing a single mass (arrows) on segment 6 with enhancement in the arterial phase (A) and wash-out on the delayed phase (B). Chest CT (C) showing no lung metastasis on initial diagnosis. Liver MRI showing a single mass (arrow) with an expansile tumor thrombus partially extending from the right portal vein to the main portal vein in the delayed arterial phase (D) of T1-weighted images. Transitional phase (E). T2-weighted image (F). Hepatobiliary phase (G). Diffusion-weighted image $(b=800)(H)$. 
The mass started to exhibit a low signal intensity in the transitional phase, with a low signal in the hepatobiliary phase and a high signal in diffusion-weighted images. Additionally, a right portal vein thrombus, which was partially expansile to the main portal vein, was observed in the delayed arterial phase (Fig. 1). The patient was staged as Modified Union for International Cancer Control stage III, T3N0M0, and Barcelona Clinic Liver Cancer (BCLC) stage BCLC C.

In the initial lab tests, HBV DNA was not detected, the $\alpha$-fetoprotein (AFP) level was above $60,500 \mathrm{ng} / \mathrm{mL}$, and the protein level induced by vitamin $\mathrm{K}$ absence-II (PIVKA-II) level was 58,341 mAU/mL. The prothrombin time was 13.2 seconds, the albumin level was $4.1 \mathrm{~g} / \mathrm{dL}$, the total bilirubin level was $0.9 \mathrm{mg} / \mathrm{dL}$, the alanine aminotransferase level was $63 \mathrm{IU} / \mathrm{L}$, and the aspartate aminotransferase level was $86 \mathrm{IU} / \mathrm{L}$. The patient did not have a history of hepatic encephalopathy or ascites. Therefore, his liver function was considered good, with a Child-Pugh score of 5, Class A. His performance status was also good, with an Eastern Cooperative Oncology Group status score of 0 .
Based on the results of clinical studies reporting that hepatic artery injection chemotherapy combined with RT was not inferior to sorafenib in patients with advanced HCC with PVTT ${ }^{5,6}$ HAIC combined with RT was performed as the first-line therapy. For HAIC, 5-FU $500 \mathrm{mg} / \mathrm{m}^{2}$ for 3 days and cisplatin $60 \mathrm{mg} / \mathrm{m}^{2}$ for 1 day were used as a fixed dose and administered twice at 4-week intervals. Radiation therapy was performed for the tumors in segment 6 and the PVTT. The total dose of 3,900 cGy was administered 13 times at 300 cGy each.

After 2 cycles of HAIC combined with RT, the portion of viable tumor exhibiting enhancement and the size of the PVTT had decreased on liver dynamic CT. However, as multiple lung metastases and new primary HCC were identified in segment 8 , the patient was considered to have progressive disease based on the modified Response Evaluation Criteria in Solid Tumors (modified RECIST) for HCC (Fig. 2).

Lenvatinib (Lenvima ${ }^{\circledR}, 12 \mathrm{mg}$ per day; Eisai Co., Ltd., Tokyo, Japan) was administered for 3 months as the first-line systemic therapy. However, the follow-up liver dynamic CT
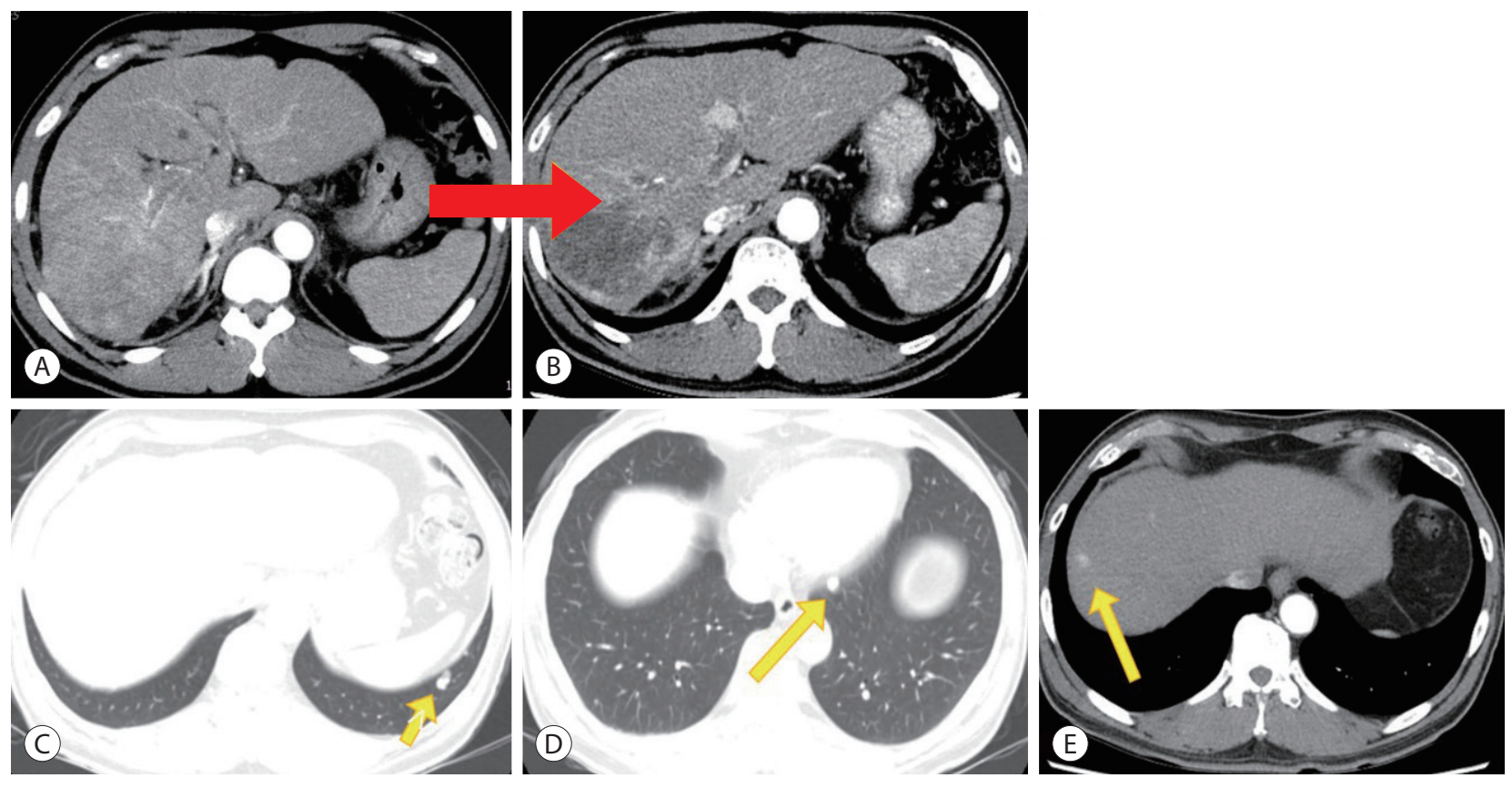

Figure 2. A change in the portal vein tumor thrombus, newly developed hepatocellular carcinoma (HCC), and lung metastasis in liver dynamic computed tomography after HAIC with radiation therapy (RT). (A, B) Shrinkage of viable tumor and portal vein tumor thrombus (red arrow) after hepatic artery infusion chemotherapy (HAIC) with RT. (C, D) Multiple lung metastases (yellow arrow) after HAIC with RT. (E) New primary HCC (yellow arrow) in segment 8 after HAIC with RT. 
showed increased arterial enhancement in a portion of the existing primary HCC, which was considered to be the viable portion of the tumor, in addition to PVTT progression. Additionally, multiple lung metastases had progressed and new HCCs were observed in several segments, which corresponded to disease progression according to modified RECIST (Fig. 3).

Therefore, sorafenib (Nexavar ${ }^{\circledR}, 400 \mathrm{mg}$ twice daily; Bayer, Leverkusen, Germany) was administered as the second-line systemic therapy. However, the patients developed grade 3 sorafenib-induced diarrhea after treatment initiation, which persisted even dose reduction. Therefore, the sorafenib treatment was terminated.

Nivolumab (Opdivo ${ }^{\circledR}, 3 \mathrm{mg} / \mathrm{kg}$ after every 2 weeks; Ono pharmaceutical, Osaka, Japan) treatment was then initiated as the third-line systemic therapy. The serum AFP and PIVKA-II levels decreased continuously from the first administration (Fig. 4A). After the third administration, the size and number of multiple lung metastases had decreased in followup CT, which corresponded to partial regression according to modified RECIST. The AFP and PIVKA-II levels decreased to $9,780 \mathrm{ng} / \mathrm{mL}$ and $87 \mathrm{mIU} / \mathrm{mL}$, respectively. Follow-up CT performed after the 18th administration of nivolumab showed that multiple pulmonary nodules had disappeared, along with the arterial enhancing HCC (Fig. 5).

Twenty-four doses of nivolumab were administered without any side effects, including impaired liver function (Fig. 4B-D). The last liver MRI showed no evidence of viable tu-
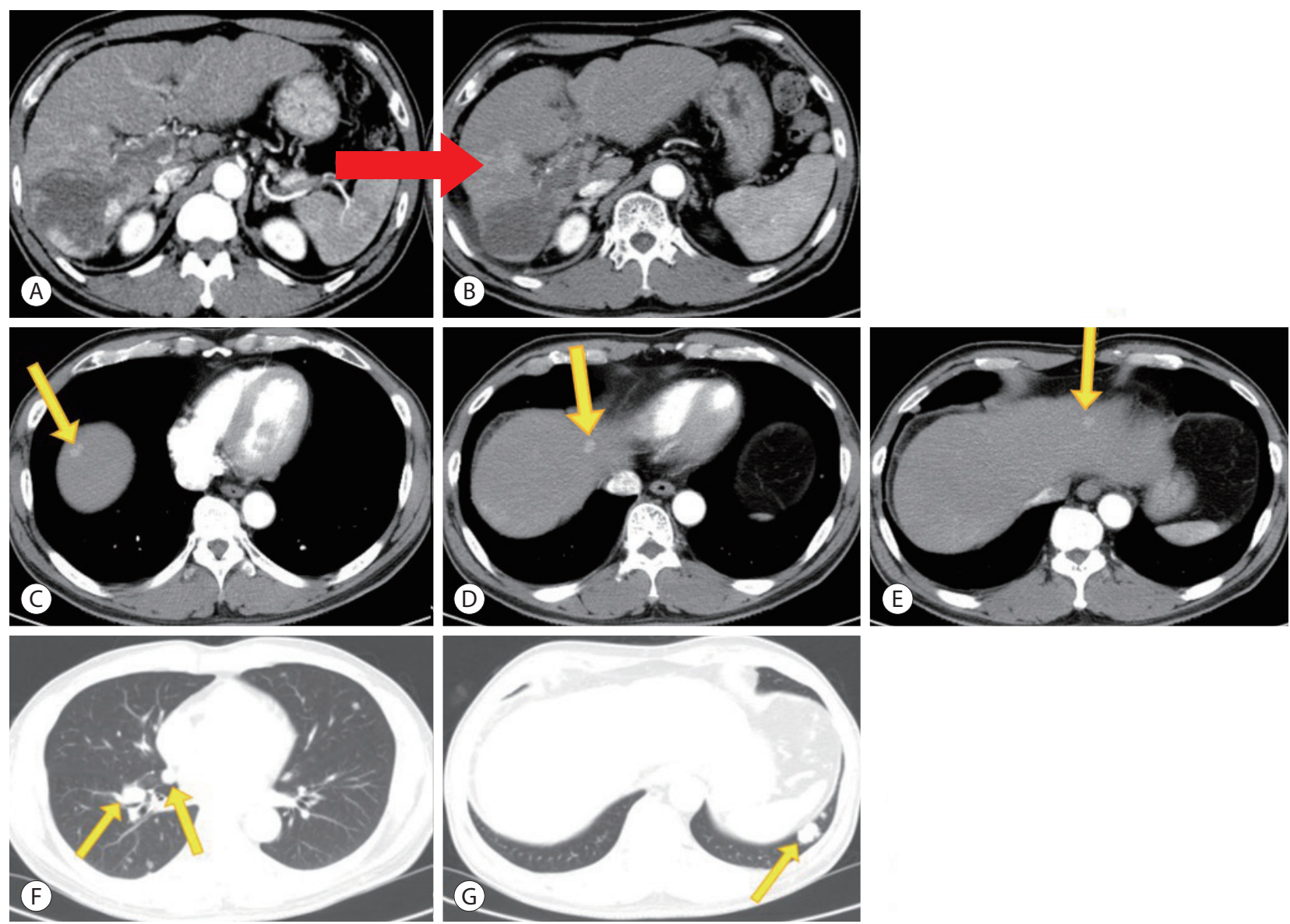

Figure 3. Progression of hepatocellular carcinoma (HCC), portal vein tumor thrombus (PVTT), and lung metastases in liver dynamic computed tomography (CT) after lenvatinib therapy. Follow-up liver dynamic CT showing increase in the arterial enhancing portion of the existing primary HCC (red arrow) and PVTT (B) compared to before lenvatinib therapy (A). Additionally, new HCCs (yellow arrows) are visible in several hepatic segments (C-E) and multiple lung metastases show progression (yellow arrows) (F, G). 

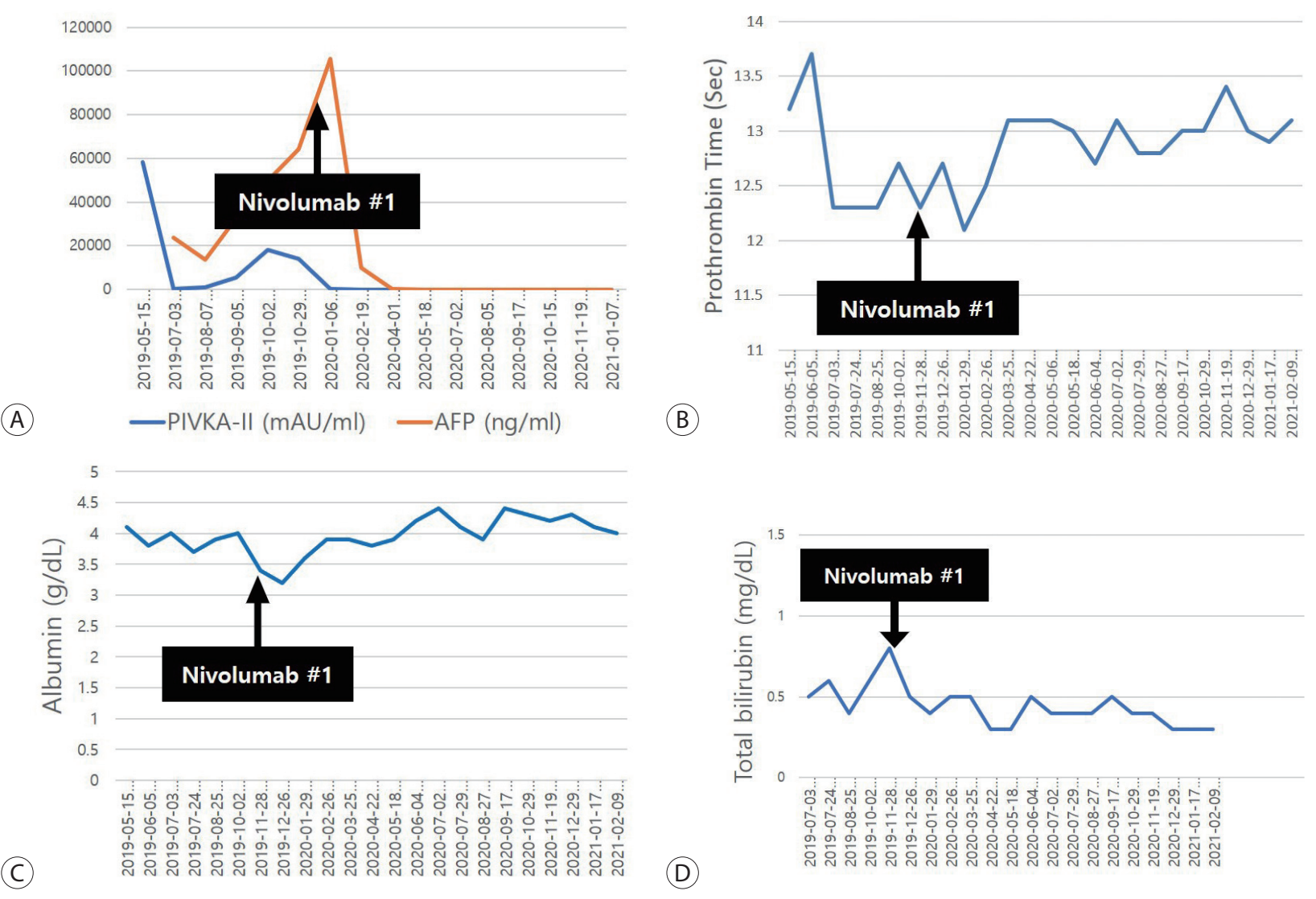

(D)

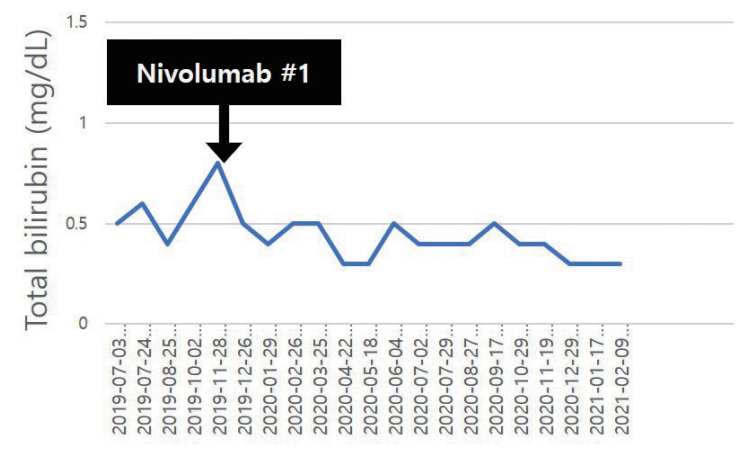

Figure 4. Changes in a-fetoprotein (AFP) and protein induced by vitamin K absence-II (PIVKA-II) levels as well as liver function during nivolumab therapy. Serum AFP and PIVKA-II levels decreased continuously from the first administration of nivolumab. (A-D) Preserved liver function during nivolumab therapy.

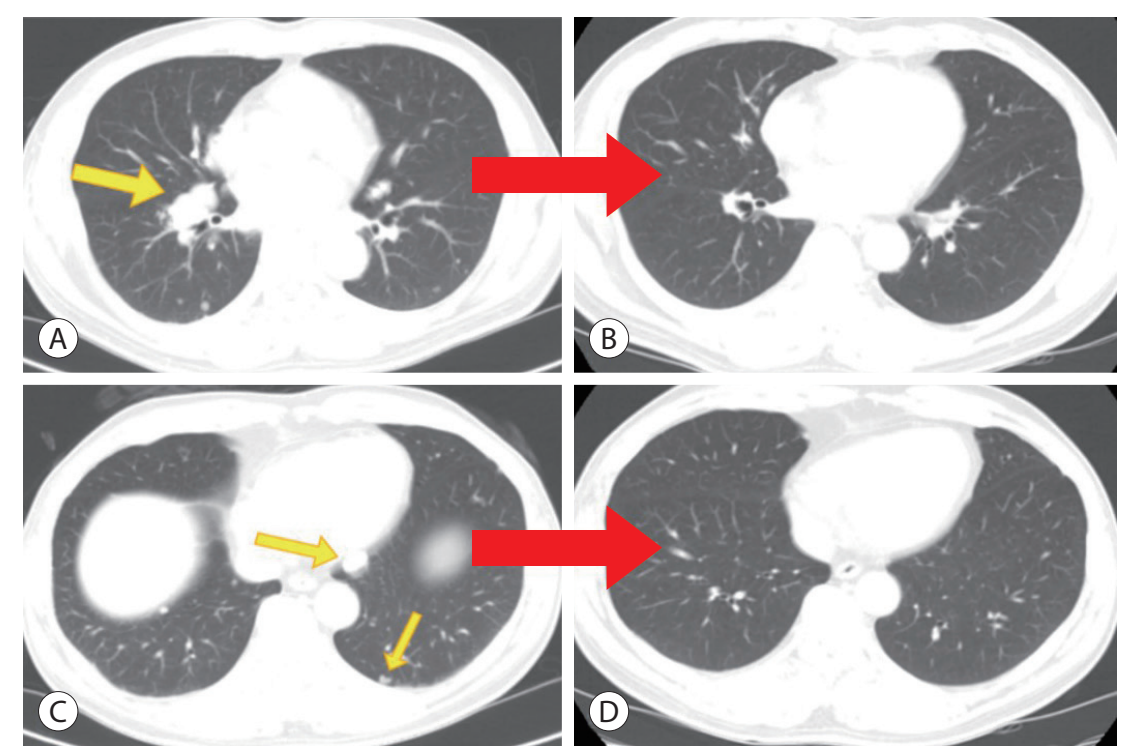

Figure 5. Complete disappearance of multiple lung metastases (arrows) on chest computed tomography after the 18th nivolumab treatment. Multiple pulmonary nodules disappeared after the 18th administration of nivolumab (B, D) compared to before nivolumab therapy ( $A, C)$. 

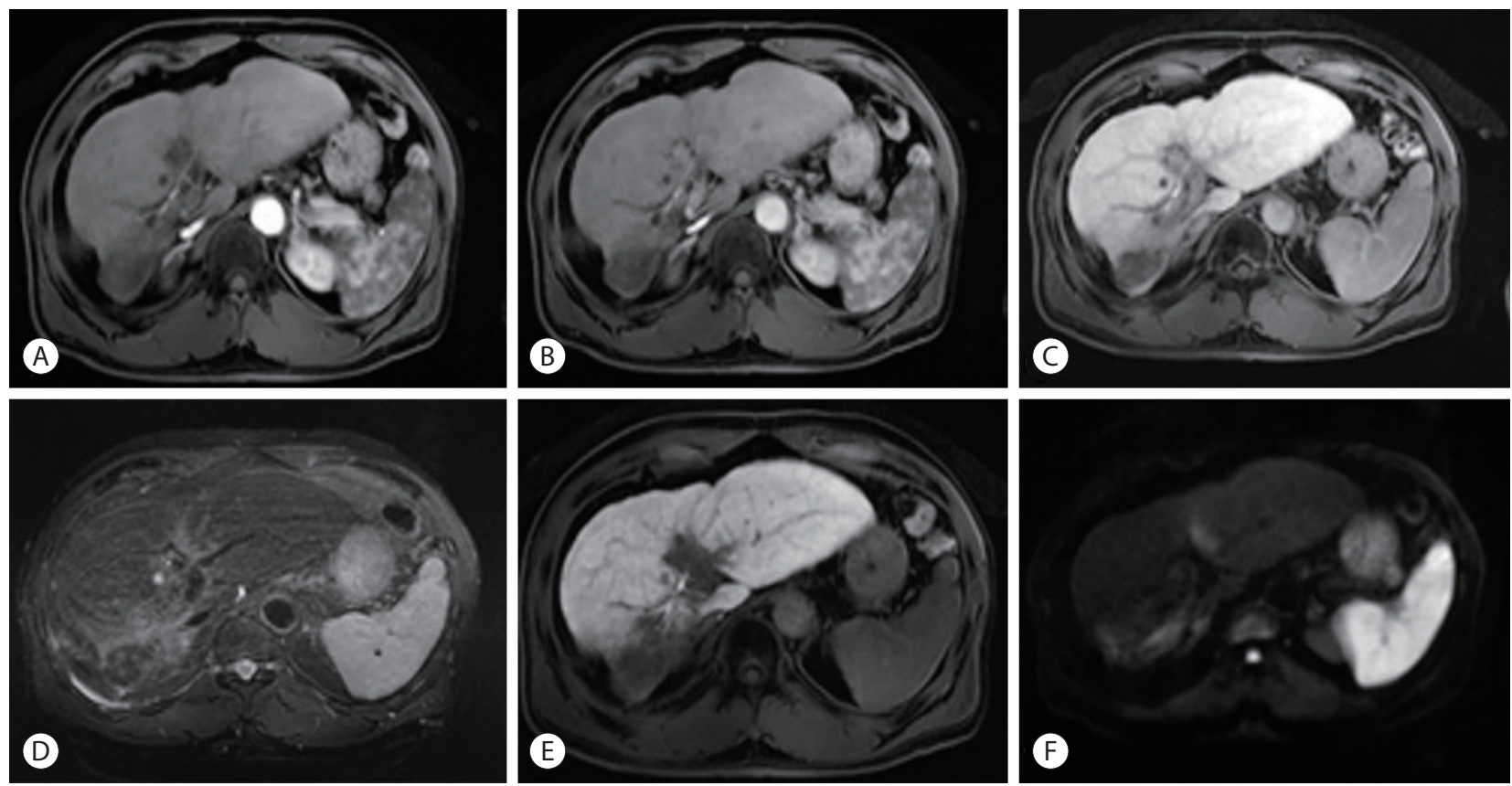

Figure 6. Liver magnetic resonance imaging after the 24th nivolumab treatment, showing no evidence of viable tumors exhibiting arterial enhancement in the liver and contracted expansile portal vein tumor thrombus. (A) T1-weighted image, hepatic arterial phase. (B) Portal venous phase. (C) Delayed phase. (D) T2-weighted image. (E) Hepatobiliary phase. (F) Diffusion-weighted image ( $b=800)$.

mors exhibiting arterial enhancement in the liver. The expansile PVTT had also contracted and all multiple pulmonary nodules had disappeared (Fig. 6). The serum AFP and PIVKA-II levels were also maintained at normal levels.

\section{Ethics statement}

The Institutional Review Board of Ajou University waived the requirement for ethics approval and informed consent (IRB number: AJIRB-MED-EXP-21-379).

\section{DISCUSSION}

HCC with PVTT occurs in $44.0-62.2 \%$ of cases ${ }^{7}$ and often causes distant metastasis. ${ }^{8}$ The lung is the most common organ for HCC metastasis, accounting for 51\% of all extrahepatic metastases. ${ }^{9}$ Patients with PVTT or extrahepatic metastases are classified as BCLC stage $\mathrm{C}$ and are considered potential candidates for systemic therapy. ${ }^{10}$

Based on the underlying feature of the cirrhotic liver; namely, an immunosuppressive environment that protects cancer cells from being recognized by the immune system, the immunotherapies like ICIs provide a strong rationale for HCC treatment. Immune checkpoints normally prevent the activation of uncontrolled autologous immunity by suppressing $\mathrm{T}$ cell activation. The overexpression of immune checkpoint molecules by cancer cells is a critical mechanism through which tumor cells evade immune surveillance. ${ }^{11}$ PD-1 is an immunosuppressive receptor expressed on activated $\mathrm{T}$ cells, $\mathrm{B}$ cells, NK cells, regulatory T cells (Tregs), myeloid-derived suppressor cells, and dendritic cells. ICIs block the inhibitory immune checkpoints and ultimately trigger an immune response targeting tumor tissue. ${ }^{11,12}$

Multiple ongoing phase I-III clinical trials are evaluating ICI monotherapy for the treatment of advanced HCC. ${ }^{13,14}$ Several clinical studies are evaluating nivolumab as monotherapy and combination therapy for advanced HCC. ${ }^{15}$ CheckMate-459, a randomized, multi-center phase III controlled trial, evaluated nivolumab as the first-line treatment for advanced HCC. However, nivolumab did not reach statistical significance for overall survival compared to sorafenib, despite exhibiting the clinical benefits of improved objective response rate, long-time disease control, fewer adverse 
events, and survival benefit. ${ }^{16}$ Only a subset of patients with advanced HCC experience clinical benefits through TKIs or ICIs therapy. However, several cases of CR from advanced HCC following the administration of nivolumab have been reported. ${ }^{17-19}$ As nivolumab was approved as second-line therapy after sorafenib failure in advanced HCC patients, nivolumab was administered in two cases with advanced HCC with preserved liver function after sorafenib failure. Another case in a patient with decompensated cirrhosis achieved CR after nivolumab as the first-line treatment. ${ }^{19}$ Since the reported CR cases are diverse in terms of underlying liver disease, prior treatment, and underlying liver function, it is difficult to infer commonalities from the fragmentary clinical or laboratory characteristics. Nevertheless, there may be a common specific immunological feature among patients who achieve a CR with nivolumab. Elucidating this immunological feature may lead to the development of a potential predictive biomarker and therapeutic target to overcome ICI resistance.

The patient in the present case was treated with HAIC and TKI before nivolumab. Previous studies reported that radiotherapy and chemotherapy can exert tumor immune response. ${ }^{20}$ These therapies damage cancer cells, leading to ROS or endoplasmic reticulum stress medicated cellular stress and subsequent immunogenic cell death. This can contribute to the transformation of immunologically "cold" tumors to "hot" tumors and improve their response to ICI treatment. ${ }^{20}$ In the present case, the prior HAIC, RT, and TKI treatment may have affected the CR with nivolumab; however, this is only a case report limited to our study. Further studies are needed to identify a possible role of prior or concomitant HAIC, TKIs, and/or RT in the antitumor effects of ICI treatment in patients with advanced HCC.

Here, we report a case of CR after nivolumab therapy, which had progressed during HAIC and TKI treatment. The findings of the present case suggest that nivolumab could be used as an effective rescue therapy for patients with HCC who demonstrate disease progression after TKI treatment.

\section{Conflicts of Interest}

The authors have no conflicts of interest to disclose.

\section{Ethics Statement}

The Institutional Review Board of Ajou University waived the requirement for ethics approval and informed consent (IRB number: AJIRB-MED-EXP-21-379).

\section{Funding Statement}

No funding to declare.

\section{Data Availability}

Data sharing not applicable to this article as no datasets were generated or analyzed for this case report.

\section{ORCID}

$\begin{array}{ll}\text { Ji Eun Han } & \text { https://orcid.org/0000-0001-8208-7709 } \\ \text { Hyo Jung Cho } & \text { https://orcid.org/0000-0003-4792-8335 } \\ \text { Soon Sun Kim } & \text { https://orcid.org/0000-0002-6862-1896 } \\ \text { Jae Youn Cheong } & \text { https://orcid.org/0000-0001-6246-1783 }\end{array}$

\section{Author Contribution}

Conceptualization: JEH, HJC

Data curation: HJC, SSK, JYC

Methodology: HJC, SSK, JYC

Project administration: HJC, SSK, JYC

Writing original draft: JEH

Writing review \& editing: HJC

Approval of final manuscript: all authors.

\section{References}

1. Global Burden of Disease Liver Cancer Collaboration, Akinyemiju T, Abera S, Ahmed M, Alam N, Alemayohu MA, et al. The burden of primary liver cancer and underlying etiologies from 1990 to 2015 at the global, regional, and national level: results from the global burden of disease study 2015. JAMA Oncol 2017;3:1683-1691.

2. Kanwal F, Kramer J, Asch SM, Chayanupatkul M, Cao Y, El-Serag HB. Risk of hepatocellular cancer in HCV patients treated with direct-acting antiviral agents. Gastroenterology 2017;153:9961005.e1001.

3. Llovet JM, Kelley RK, Villanueva A, Singal AG, Pikarsky E, Roayaie $S$, et al. Hepatocellular carcinoma. Nat Rev Dis Primers 2021;7:6.

4. Yim SY, Lee JS. The genomic landscape and its clinical implications in hepatocellular carcinoma. J Liver Cancer 2019;19:97-107.

5. Choi JH, Chung WJ, Bae SH, Song DS, Song MJ, Kim YS, et al. Ran- 
domized, prospective, comparative study on the effects and safety of sorafenib vs. hepatic arterial infusion chemotherapy in patients with advanced hepatocellular carcinoma with portal vein tumor thrombosis. Cancer Chemother Pharmacol 2018;82:469-478.

6. Song DS, Song MJ, Bae SH, Chung WJ, Jang JY, Kim YS, et al. A comparative study between sorafenib and hepatic arterial infusion chemotherapy for advanced hepatocellular carcinoma with portal vein tumor thrombosis. J Gastroenterol 2015;50:445-454.

7. Guo Y, Li J, Dong P, Feng $X, X u J, Y e ~ Y$, et al. Epidemiological serosurvey of hepatitis $B$ in national disease surveillance points of Henan province in 2014. Zhonghua Yu Fang Yi Xue Za Zhi 2016:50:279-281.

8. Chan SL, Chong CC, Chan AW, Poon DM, Chok KS. Management of hepatocellular carcinoma with portal vein tumor thrombosis: Review and update at 2016. World J Gastroenterol 2016;22:72897300.

9. Katyal S, Oliver JH 3rd, Peterson MS, Ferris JV, Carr BS, Baron RL. Extrahepatic metastases of hepatocellular carcinoma. Radiology 2000;216:698-703.

10. Llovet JM, Brú C, Bruix J. Prognosis of hepatocellular carcinoma: the BCLC staging classification. Semin Liver Dis 1999;19:329-338.

11. Mohr R, Jost-Brinkmann F, Özdirik B, Lambrecht J, Hammerich L, Loosen $\mathrm{SH}$, et al. Lessons from immune checkpoint inhibitor trials in hepatocellular carcinoma. Front Immunol 2021;12:652172.

12. Ringelhan $M$, Pfister $D, O^{\prime}$ Connor $T$, Pikarsky $E$, Heikenwalder $M$. The immunology of hepatocellular carcinoma. Nat Immunol 2018;19:222-232.

13. Finn RS, Ryoo BY, Merle $P$, Kudo M, Bouattour M, Lim HY, et al. Pembrolizumab as second-line therapy in patients with advanced hepatocellular carcinoma in KEYNOTE-240: a randomized, doubleblind, phase III trial. J Clin Oncol 2020;38:193-202.

14. Qin S, Ren Z, Meng Z, Chen Z, Chai X, Xiong J, et al. Camrelizumab in patients with previously treated advanced hepatocellular carcinoma: a multicentre, open-label, parallel-group, randomised, phase 2 trial. Lancet Oncol 2020;21:571-580.

15. Cheng H, Sun G, Chen H, Li Y, Han Z, Li Y, et al. Trends in the treatment of advanced hepatocellular carcinoma: immune checkpoint blockade immunotherapy and related combination therapies. Am J Cancer Res 2019;9:1536-1545.

16. Yau T, Park JW, Finn RS, Cheng AL, Mathurin P, Edeline J, et al. LBA38_PR - CheckMate 459: A randomized, multi-center phase III study of nivolumab (NIVO) vs sorafenib (SOR) as first-line (1L) treatment in patients (pts) with advanced hepatocellular carcinoma (aHCC). Ann Oncol 2019;30:v874-v875.

17. Kim J, Chang JW, Park JY. Nivolumab for advanced hepatocellular carcinoma with multiple lung metastases after sorafenib failure. J Liver Cancer 2020;20:72-77.

18. Zhu SG, Li HB, Yuan ZN, Liu W, Yang Q, Cheng Y, et al. Achievement of complete response to nivolumab in a patient with advanced sarcomatoid hepatocellular carcinoma: a case report. World J Gastrointest Oncol 2020;12:1209-1215.

19. Trojan J, Sarrazin C. Complete response of hepatocellular carcinoma in a patient with end-stage liver disease treated with nivolumab: whishful thinking or possible? Am J Gastroenterol 2016;111:1208-1209.

20. Liu YT, Sun ZJ. Turning cold tumors into hot tumors by improving T-cell infiltration. Theranostics 2021;11:5365-5386. 